Ann. Sci. forest., 1971, 28 (2), 185-194.

\title{
HÉRITABILITÉ DE LA DENSITÉ DU BOIS DE SAPIN PECTINÉ
}

\author{
H. POLGE \\ avec la collaboration technique de S. Garros et P. Michel \\ Station de Recherches sur la Qualité des Bois, \\ Centre national de Recherches forestières, I.N.R.A., \\ 54 - Champenoux
}

\section{RÉSUMÉ}

L'héritabilité de la densité du bois de Sapin pectiné est étudiée à l'âge de quatre ans sur 20 familles issues de pollinisation libre et 10 descendants par famille.

Par étude de la régression parents-descendants, la valeur d'héritabilité trouvée est de 0,33 , et le coefficient de corrélation de 0,56 ; l'analyse de la variance entre familles et à l'intérieur des familles conduit à une valeur d'héritabilité de 0,22 .

Ces valeurs d'héritabilité relativement faibles sont probablement due à l'échantillonnage (très faibles dimensions des plants — de 3 à $9 \mathrm{~mm}$ de diamètre _, prélèvement des échantillons dans une seule répétition).

Il existe une liaison significative entre la dureté des arbres-mères mesurée sur pied à l'aide d'un torsiomètre et la densité des semis.

En comparant entre elles les densités des semis issus de deux générations successives du même peuplement, on observe une baisse significative de la densité en passant des premiers aux seconds, ce qui est sans doute à rapprocher du débourrement plus tardif de ceux-ci.

\section{1. - INTRODUCTION}

Dans toutes les études de génétique quantitative faites jusqu'à présent sur des arbres forestiers, la densité du bois apparaît comme un critère fortement héréditaire, son héritabilité étant en général nettement supérieure à celle des autres caractéristiques technologiques (longueur de fibres, rectitude du fil du bois...), et plus encore à celle des largeurs de cernes.

Les références les plus nombreuses et les plus anciennes relatives à l'héritabilité au sens strict de la densité du bois concernent les pins du Sud des Etats-Unis : 
Pour Pinus elliotti, SQuillace et al. (1962) ont trouvé, pour des sujets de 14 ans, une héritabilité de 0,21 dans le cas de descendances obtenues par fécondation libre, et de 0,56 en pollinisation contrôlée.

Pour Pinus taeda, Stonecypher et al. (1964) ont calculé, sur 100 descendances issues de pollinisation libre, une héritabilité de 0,55 à deux ans. VAN BuiJTEnen (1962) a, pour sa part, mentionné, pour des semis de deux ans également, mais obtenues en fécondation contrôlée, des héritabilités de 0,37 et de 0,49. GogGans enfin (1962) a, dans deux expériences limitées malheureusement aux descendances issues de pollinisation libre de 6 arbres-mères, d'une part, et 8 arbres-mères, de l'autre, trouvé, à 5 ans, des héritabilités très élevées : de 0,76 et de 0,87 respectivement.

En France, des études d'héritabilité ont été faites sur des Pins maritimes âgés de 4 ans (Polge et Illy, 1968); elles ne portaient que sur 9 familles différentes obtenues par fécondation libre; les valeurs d'héritabilité, calculées par analyse de variance, entre les descendances, étaient de 0,56 pour la densité du côté Est, 0,49 pour la densité Ouest, et 0,75 pour la densité moyenne totale ; par analyse de la régression parents-descendants, on a obtenu une héritabilité de 0,56 pour la densité totale du côté Est, de 0,66 entre la densité totale des descendants du côté Ouest et la densité moyenne sur 20 cernes du côté Ouest des arbres-mères, et, enfin, de 0,72 pour la densité moyenne totale, la très grande similitude des résultats trouvés par l'une ou l'autre méthode ne pouvant que résulter de coïncidences heureuses eu égard au faible nombre de familles.

En ce qui concerne la densité du bois de Sapin pectiné, aucune étude génétique ne paraît lui avoir été consacrée jusqu'ici, et il était donc intéressant de profiter d'une expérience entreprise par la Station d'Amélioration des Arbres Forestiers, dont les premiers résultats ont été publiés par Arbez et Millier (1970), pour voir si, comme pour les autres espèces résineuses précédemment étudiées, cette caractéristique était, ou non, fortement influencée par le patrimoine héréditaire. Tel est l'objet du présent travail.

\section{2. - MATÉRIEL ET MÉTHOdE}

\section{1. - Dispositif expérimental}

Le dispositif complet comprenait, rappelons-le :

- 20 arbres parfaitement repérés sur le terrain dans la forêt de la Bétouze d'Arques,

- 20 arbres non repérés du peuplement du Rialsesse présumé issu du précédent par régénération naturelle,

- des descendances maternelles de chacun de ces 40 arbres-mères comparées entre elles au sein de deux dispositifs expérimentaux équilibrés, identiques mais distincts, portant au total sur 6 répétitions de 10 semis pour chaque descendance.

Il est ainsi possible de faire des calculs d'héritabilité, à la fois par analyse de variance dans les jeunes plants des diverses familles, et par étude de régression parents-descendants pour le peuplement de la Bétouze d'Arques, d'une part, et de comparer entre elles les descendances des deux générations successives que forment la forêt de la Bétouze d'Arques et la forêt du Rialsesse, d'autre part. 


\section{2. - Matériel d'étude pour les arbres-mères}

Il a consisté en carottes de sondage de $5 \mathrm{~mm}$ de diamètre prélevées à hauteur d'homme suivant une direction correspondant en principe aux courbes de niveau, pour éviter la zone de bois de compression qui, du fait de la déclivité du terrain, se trouve en général concentrée sur le côté aval des trones.

Les couples de torsion nécessaires pour enfoncer la tarière à $3 \mathrm{~cm}$ à l'intérieur du bois ont été mesurés suivant une méthode décrite par ailleurs (Polge et Keller, 1970).

Les carottes obtenues ont été coupées en deux morceaux, l'un formé par les 50 derniers cernes et représentant le bois adulte, lautre correspondant au reste des échantillons et comprenant donc le bois produit pendant la période juvénile de croissance des arbres en cause.

La densité du bois a été déterminée, pour tous les arbres échantillonnés, séparément sur les deux fragments ainsi délimités par une variante de la méthode de la saturation intégrale, dans laquelle le poids maximum est obtenu par trempage pendant 48 heures dans de l'eau bouillante, suivi d'une période de refroidissement de 12 heures.

La partie formée par les 50 cernes les plus récents a été radiographiée en vue d'études de structure fine des accroissements annuels, en utilisant une distance source-film de $2,50 \mathrm{~m}$, une tension accélératrice de $15 \mathrm{kV}$, une intensité du flux électronique de $20 \mathrm{~mA}$, et un temps d'exposition de $5 \mathrm{mn}$.

Lors des opérations de prélèvement, un des arbres-mères de l'expérience, dont les graines avaient été préalablement récoitées, était exploité, en sorte que les échantillons pour étude de densité n'ont pu être recueillis que sur 19 arbres. De plus, la carotte de sondage de l'arbre n" 6 s'est trouvée, malgré les précautions prises, dans la zone de bois de compression et a donc dû être éliminée. On a, par suite, adopté pour cet arbre, comme valeur de densité, la moyenne obtenue à partir de deux carottes de bois normal prises à $30^{\circ}$ de part et d'autre de la direction opposée au premier prélèvement.

\section{3. - Matériel d'étude pour les descendants}

Les plants d'une des répétitions, c'est-à-dire 10 individus par famille, ont été coupés à l'âge de quatre ans en vue de la détermination, à un stade très précoce, de la densité du bois.

Compte tenu de la très faibie dimension des échantillons (les diamètres au collet sous écorce étaient compris entre 3 et $9 \mathrm{~mm}$ ), des précautions toutes particulières ont dû être prises pour réduire dans toute la mesure du possible l'erreur expérimentale qui était à craindre au cas particulier. A cet effet, trois échantillons répertoriés A, B et C ont été découpés dans chaque tige à partir du point où l'influence perturbatrice du collet cesse de se manifester, c'est-à-dire à 2 ou $3 \mathrm{~mm}$ au-dessus de celui-ci. Le premier échantillon (référence A) était constitué par un fragment de tige de longueur $35 \mathrm{~mm}$ dont la densité a été calculée à partir du poids anhydre et du volume saturé mesuré au voluménomètre à mercure. Les échantillons B étaient des rondelles d'épaisseur rigoureusement uniforme et égale à $2,2 \mathrm{~mm}$; ils ont été radiographiés en utilisant une distance source-film de $2,50 \mathrm{~m}$, une tension accélératrice de $10,5 \mathrm{kV}$, une intensité du flux électronique de $20 \mathrm{~mA}$ et une durée d'exposition de 4 heures. Les images obtenues ont servi, d'une part à des études fines de structure des accroissements annuels, et d'autre part au contrôle optique des densités déterminźes sur les autres échantillons. Immédiatement au-dessus de la rondelle $\mathrm{B}$, une autre rondelle de $5 \mathrm{~mm}$ d'épaisseur a été prélevée (référence C) et sa densité calculée par la méthode de saturation intégrale déjà utilisée pour leз carottes des arbres-mères.

Les déterminations de densité des échantilions $\mathrm{A}$ et $\mathrm{C}$ ont été refaites lorsqu'elles étaient trop différentes de ce que laissait prévoir le noircissement des images radiographiques de $\mathbf{B}$, puis, après ces vérifications, on a adopté comme valeur définitive de densité la moyenne de celles obtenues à partir des échantillons A et C. Cette façon de procéder se justifie par le fait qu'il n'y a à priori aucune raison pour préférer l'une ou l'autre des deux méthodes employées :

La taille supérieure des fragments de tige A permettait en principe de réduire les erreurs relatives de mesure, mais en contrepartie la détermination des volumes au voluménomètre à mercure manque de précision; la méthode de saturation intégrale utilisée pour les échantillons $C$ ne fait appel qu'à des pesées pour lesquelles il existe maintenant des balances qui donnent couramment le $1 / 10^{\circ}$ de miligramme: mais la saturation ne peut être obtenue en toute certitude que si l'épaisseur des échantillons dans le sens du fil du bois ne dépasse pas quelques millimètres; de la sorte, la taille des échantillons $\mathrm{C}$ se trouvait forcément limitée ; ils ne représentaient donc qu'une très faible partie de la tige, d'où l'inopportunité qu'il y avait à leur attribuer, pour la détermination de la densité moyenne, un poids supérieur à celui des densités $\mathrm{A}$. 


\section{3. - RÉSULTATS}

\section{1. - Comparaison globale des descendances de la Bétouze d'Arques et $d u$ Rialsesse}

Elle a porté sur l'ensemble des 200 plants issus du peuplement primaire (Bétouzc d'Arques) et des 200 plants issus de la génération suivante (Rialsesse).

On a trouvé les valeurs moyennes suivantes :

- Densité moyenne descendances Bétouze d'Arques: 554,1 g/ $\mathrm{dm}^{3}$,

- Densité moyenne descendances Rialsesse: $538,2 \mathrm{~g} / \mathrm{dm}^{3}$.

On observe donc une baisse de densité de $16 \mathrm{~g} / \mathrm{dm}^{3}$, ou encore en valeur relative de $3 \%$, et ie test de comparaison des moyennes $t$ est égal à 5,19 , c'est-à-dire est très significatif.

Cette première observation est intéressante si l'on se rappelle (ARBEZ et MiLlier, 1970, op. cit.) que, vraisemblablement par suite de sélection naturelle intervenue au stade juvénile au détriment des arbres à débourrement précoce dans le peuplement du Rialsesse, les descendants des arbres-mères qui en sont issus sont significativement plus tardifs que ceux des arbres-mères de la Bétouze d'Arques. Ils ont certes aussi une croissance plus rapide, aussi bien en hauteur qu'en diamètre, et l'on peut admettre que, pour le Sapin pectiné, il existe une liaison négative entre largeur et densité des accroissements annuels qui expliquerait la perte de densité observée en passant de la descendance Bétouze d'Arques à la descendance Rialsesse.

Mais on doit rappeler que ThIERCElin (1970) a également trouvé que des Epicéas adultes à débourrement tardif avaient une densité du bois significativement inférieure à celle d'arbres précoces du même peuplement, sans qu'il existe de différences entre eux au point de vue largeur des cernes.

De même, dans une comparaison de provenances d'Epicéas communs (LACAZE et Polge, 1970), la densité du bois et la tardiveté du débourrement étaient liées négativement de façon très significative $(r=-0,68 * * *$ avec 15 degrés de liberté), alors que les liaisons n'étaient significatives, ni entre la densité du bois et la hauteur, ni entre la densité du bois et le diamètre.

Il semblerait donc que l'antinomie entre une densité élevée du bois et la tardiveté du débourrement puisse être un phénomène assez général, indépendamment des liaisons qui peuvent exister entre ceile-ci et les critères de vigueur.

\section{2. - Héritabilité de la densité du bois}

\subsection{Calcul par analyse de la régression parents-descendants.}

Sur la figure 1 sont représentées, sous forme graphique, les relations entre den. sités des arbres-mères et moyennes des densités des descendants, avec les deux droite: de régression correspondantes.

S'agissant de semis obtenus par fécondation libre, on a comme valeur d'héri. tabilité $h^{2}$ :

$$
h^{2}=2 b
$$

$b$ étant le coefficient de la régression descendant/arbre-mère.

Ici donc:

$$
h^{2}=2 \times 0.164=0,33
$$


Cette valeur d'héritabilité n'est pas très élevée, mais elle est cependant remarquable si l'on tient compte de la taille extrêmement réduite des échantillons utilisés pour la détermination des densités sur les descendants, et des risques d'erreur qui en résultaient, malgré les précautions prises.

Au reste, lorsque la population des parents et celle des descendants ont été élevées dans des conditions très différentes, il est recommandé (HANSON et RoBINSON, 1963) d'utiliser, non pas le coefficient de la régression, mais le coefficient de corré-

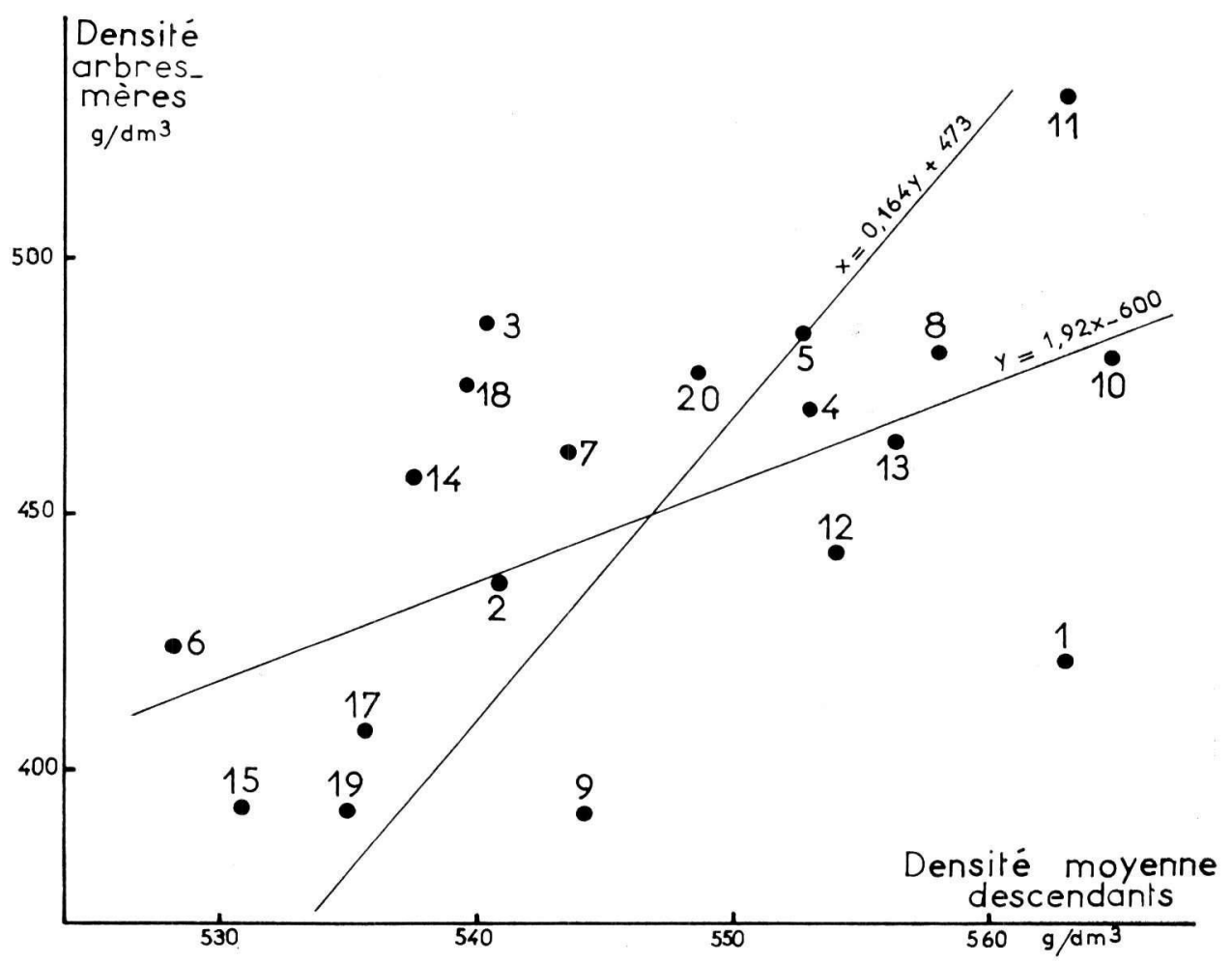

FIG. 1. - Relations entre densité des arbres-mères et densité des descendants

FIG. 1. - Reationship between mother-tree and progeny specific gravities

lation $r$, qui tient mieux compte des éventuelles différences de variance et de moyenne entre les deux populations, qui sont au cas particulier relativement importantes.

Ici, le coefficient de corrélation est très significatif et se chiffre à 0,56.

Cette liaison particulièrement étroite montre, non seulement que la densité du bois dans le jeune âge est sous une forte dépendance de l'hérédité, mais encore que la densité au stade adulte est très liée à la densité juvénile.

On peut en avoir une confirmation dans les résultats de l'étude de la corrélation qui a été faite entre la densité des 50 derniers cernes côté écorce et celle du reste des carottes; on ne disposait en fait que de 14 couples de mesures, 5 échantillons s'étant trouvés, à très peu près, limités aux cernes périphériques. 
On a obtenu les valeurs moyennes suivantes:

- densité côté écorce : 449,2 g/dm:3,

- densité reste des carottes : 439,8 g/ dm:

ce qui permet de vérifier l'existence d'un gradient positif de la moelle vers l'écorce,

- et un coefficient de corrélation très significatif de 0,80 qui confirme l'étroitesse des liaisons juvénile-adulte pour la densité du bois évoquée plus haut.

Une illustration des relations étroites qui existent entre la densité des arbresmères et la densité dé leurs descendants peut être vue en figure 2 qui regroupe, pour

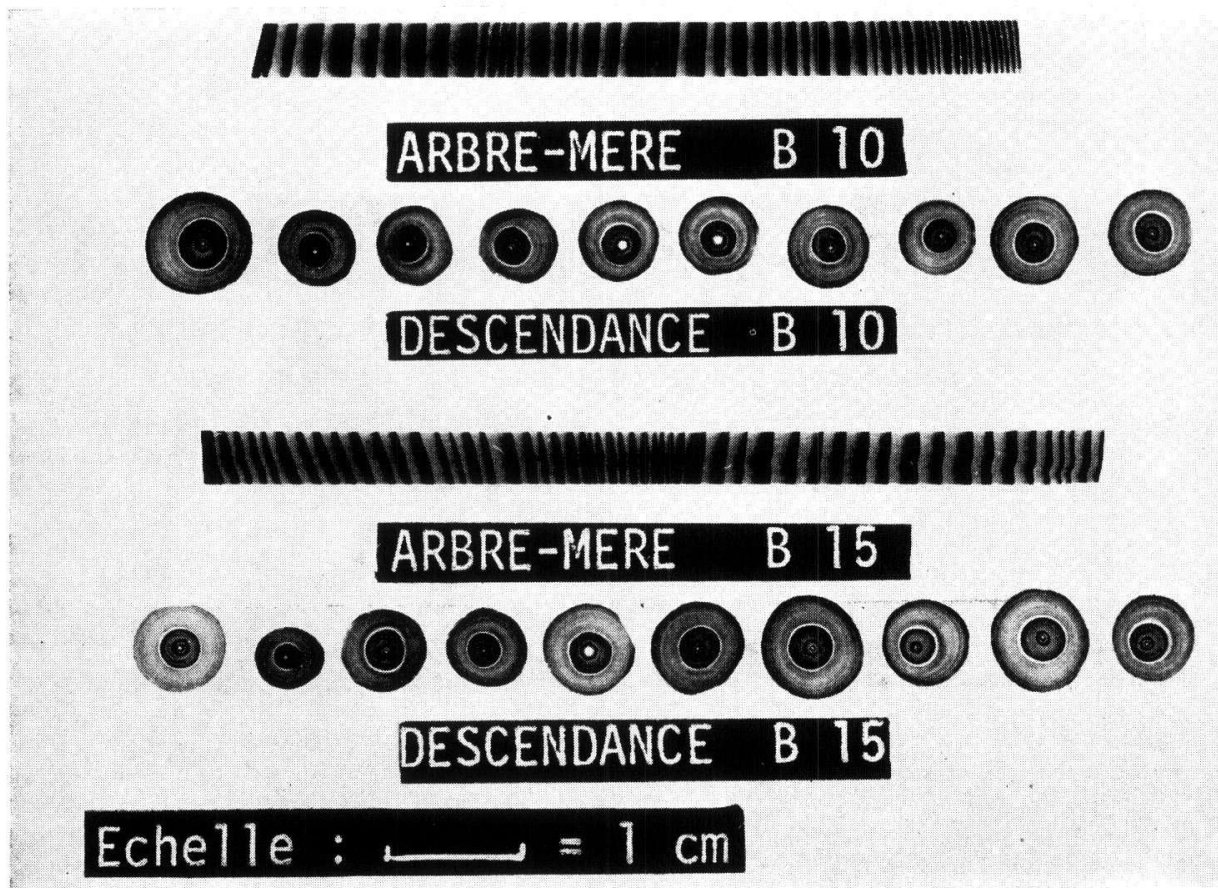

FIG. 2. - L'héritabilité en image. Positifs de radiographies des carottes tirées des arbres-mères et des rondelles de $2 \mathrm{~mm}$ d'épaisseur des descendants pour deux familles (agrandis 2 fois)

Fig. 2. - Illustration of the inheritance. Positive prints of X-ray photographs taken from the increment cores for the mother-trees and from the $2 \mathrm{~mm}$ slices for the progenies of 2 families (enlarged 2 times)

les familles B 10 et B 15, les positifs de la radiographie de la carotte de l'arbre-mère, d'une part, et des 10 rondelles de la descendance, d'autre part; s'agissant d'un cliché inversé, l'image est d'autant plus foncée que le bois est plus dense.

La densité plus élevée de la famille B 10 apparaît ainsi très nettement pour les deux générations successives; on se rend compte aussi que si la famille B 15 a un bois moins dense, elle le doit surtout à son bois initial qui, sur la figure, est beaucoup plus clair que celui de la famille B 10, qu'il s'agisse de l'arbre-mère ou de la grande majorité des descendants; enfin, le seul examen du noircissement du cliché fait apparaître une forte liaison entre les densités du dernier et de l'avant-dernier cerne sur les jeunes semis. 


\subsection{Calcul par analyse de variance sur les descendants.}

On sait que, dans des familles de demi-frères, l'héritabilité $h^{2}$ est égale à quatre fois le coefficient de corrélation intraclasse $t$, ce dernier se définissant lui-même comme le rapport de la variance entre familles à la variance totale.

Ici, les résultats de l'analyse de variance se présentent comme suit :

\begin{tabular}{l|c|c|c}
\hline $\begin{array}{c}\text { Source de } \\
\text { variation }\end{array}$ & Degrés de liberté & Carrés moyens & $\begin{array}{c}\text { Décomposition } \\
\text { des carrés moyens }\end{array}$ \\
\hline Familles $\quad \ldots \ldots \ldots \ldots$ & 19 & 1182,23 & $\sigma_{\mathrm{F}}^{2}+\sigma^{2}{ }_{\mathrm{F}}$ \\
Erreur $\ldots \ldots \ldots \ldots \ldots$ & 180 & 749,35 & $\sigma^{2}{ }_{\mathrm{W}}$ \\
\hline
\end{tabular}

si $\sigma^{2}{ }_{\mathrm{F}}=$ variance entre familles

et $\sigma_{\mathrm{E}}^{2}=$ variance de l'erreur (variance résiduelle des individus à l'intérieur des familles).

Le test $\mathrm{F}$, rapport des carrés moyens, se chiffre à 1,577 ; la valeur la plus voisine tirée de la table de Snedecor est celle qui correspond à $20 \mathrm{DL}$ pour le traitement et un nombre infini de DL pour l'erreur et qui se chiffre à 1,57. On peut donc dire qu'il existe des différences de densité entre familles significatives à un seuil de probabilité compris entre $10 \%$ et $5 \%$, mais très voisin de celui-ci.

De la décomposition des carrés moyens, on tire :

$$
\begin{aligned}
\sigma^{2}{ }_{\mathrm{F}} & =43,3 \\
\text { et } \sigma_{\mathrm{E}}+\sigma_{\mathrm{F}}^{2} & =792,6,
\end{aligned}
$$

d'où un coefficient intraclasse de 0,054 , et une héritabilité :

$$
h^{2}=0,22 \text {. }
$$

\section{3. - Estimation de la densilé des descendants}

à partir du couple de torsion mesuré sur les arbres-mères

Une analyse des régressions qui lient ces deux caractéristiques a été faite pour 18 familles (le couple de torsion de l'arbre-mère $6 \mathrm{~B}$ n'ayant été mesuré que sur la carotte de bois de compression éliminée par la suite).

Une représentation graphique des résultats est donnée en figure 3; la liaison peut être considérée comme assez étroite, compte tenu de l'hétérogénéité des matériels en cause.

Le coefficient de corrélation de 0,47 est significatif au seuil de $5 \%$.

On peut observer que la sélection en forêt de l'arbre le plus dur aurait donné la deuxième meilleure descendance au point de vue densité du bois; en revanche, à l'arbre le moins dur correspond, soit la deuxième, soit la neuvième moins bonne descendance.

Cette liaison ne doit pas surprendre lorsqu'on connaît la corrélation étroite qui lie la densité au couple de torsion mesuré lors du prélèvement (le coefficient de corré- 
lation calculé ici entre ces deux données pour les 19 arbres-mères, y compris donc la carotte de bois de compression de l'arbre $\mathrm{n}^{\circ} 6 \mathrm{~B}$, se chiffre à 0,89 ).

Cette expérience confirme ainsi la possibilité qu'il y a de sélectionner in situ, pour la densité du bois à l'aide du torsiomètre, les écarts, certes notables quelquefois, que l'on observe par rapport à la droite de régression de $\mathrm{X}$ en $\mathrm{Y}$, s'expliquant assez aisément par l'extrême difficulté des déterminations de densité sur les semis de très petite taille de certaines descendances.

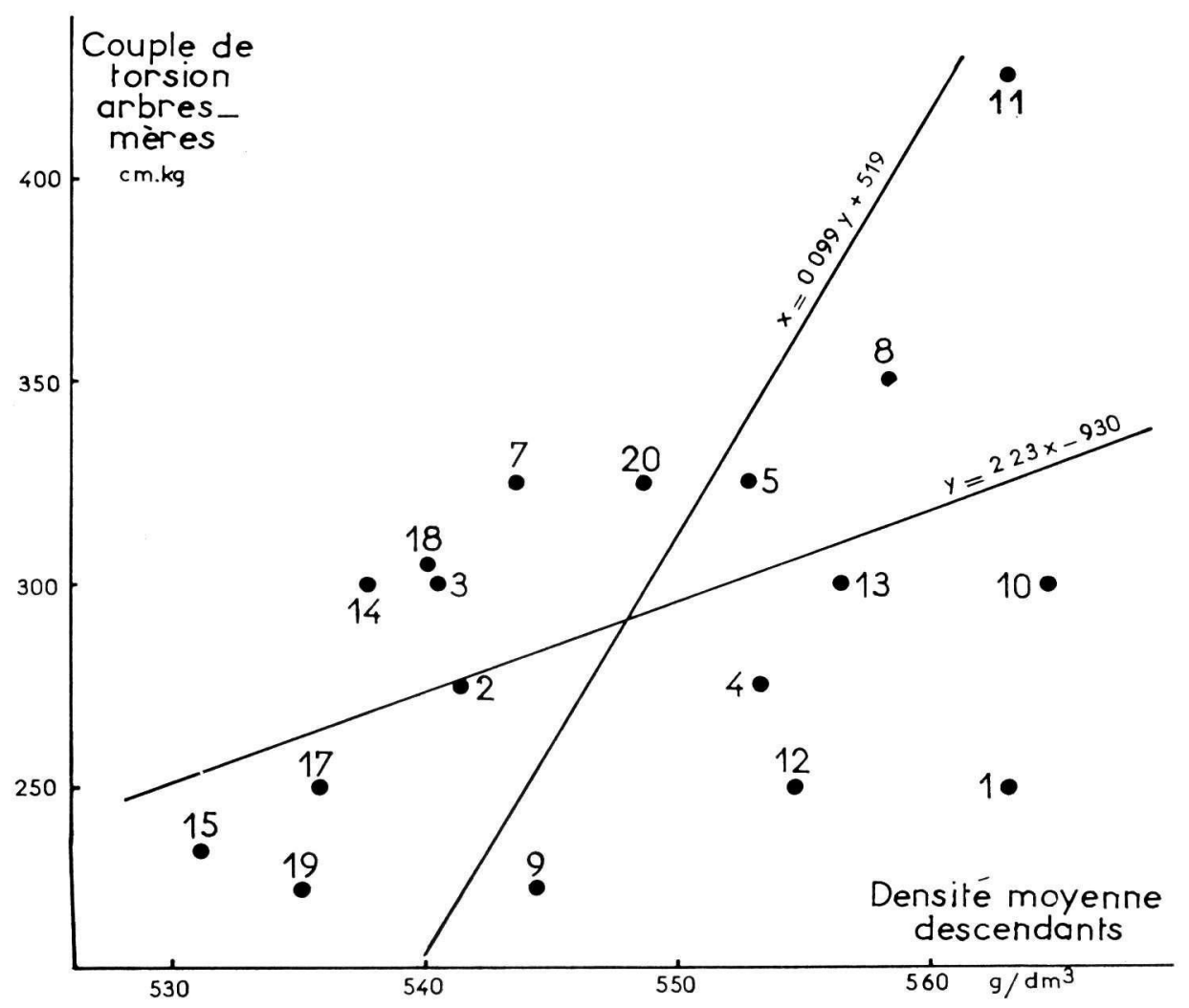

Fig. 3. - Relations entre couple de torsion des arbres-mères et des descendants

Fig. 3. - Relationship between mother tree torsion torque and progeny specific gravity

\section{4. - CONCLUSION}

Les valeurs calculées ici pour l'héritabilité du bois peuvent paraître assez faibles par comparaison avec celles obtenues précédemment sur d'autres essences résineuses. Cet état de choses ne résulte sans doute pas de quelque caractéristique fondamentale propre à l'espèce en cause et doit beaucoup plus vraisemblablement être attribué à l'échantillonnage :

Pour les arbres-mères, une seule carotte a été prélevée, en sorte qu'il n'est pas exclu que pour certains la densité ait été faussée par la présence d'un peu de bois de 
rompression moins caractérisé que dans l'arbre B 6, et, pour cette raison, non éliminé pour la détermination des densités. En outre, un effet de l'environnement reste possible, ces arbres étant échelonnés tout au long d'un versant à pente relativement forte.

Pour les descendants, les défauts de l'échantillonnage sont encore plus évidents: une seule répétition a été utilisée, d'où des risques possibles d'erreurs dans l'estimation des moyennes de familles; en outre, la taille extrêmement réduite de certains échantillons, à laquelle il a déjà été fait allusion à plusieurs reprises, et la présence également de bois de compression sur d'assez nombreux plants, se traduisant notamment par des excentricités de la moelle comme on peut en voir sur la figure 2, n'ont pu qu'augmenter considérablement la somme des carrés résiduelle dans l'analyse de variance faite sur les descendants.

Les effets probables des diverses sources de variations, autres que la famille, sur les déterminations de densité ainsi faites, peuvent également expliquer le fait que l'héritabilité calculée par analyse de variance soit inférieure à celle obtenue à partir du coefficient de régression descendant/parent, puisque la variance de l'erreur intervient directement au dénominateur du coefficient de corrélation intraclasse.

On peut par suite considérer que la valeur plus élevée d'héritabilité résultant de la comparaison entre arbres-mères et descendants correspond à un minimum, et ce d'autant qu'un calcul identique fait à partir du coefficient de corrélation aurait pu être parfaitement justifié ; il aurait abouti à une valeur de $h^{2}$ beaucoup plus élevée : en fait supérieure à 1 , ce qui est anormal, mais peut s'expliquer par le petit nombre de familles utilisées.

La densité du bois peut donc être provisoirement considérée comme fortement héréditaire chez le Sapin pectiné, mais cette étude demande à être reprise avec un plus grand nombre de familles, plusieurs échantillons par arbre-mère, et en ce qui concerne les descendants, avec des sujets sensiblement plus âgés, éduqués en pépinière à plus fort espacement, et prélevés dans plusieurs répétitions différentes.

Reçu pour publication en février 1971.

\section{SUMMARY \\ INHERITANCE OF SPECIFIC GRAVITY IN FOUR-YEAR-OLD SEEDLINGS \\ OF SILVER FIR}

The inheritance of wood specific gravity in 4-year-old seedlings of Silver Fir is studied from 20 open-pollinated families and 10 seedlings per family.

For mother trees, the samples were $5 \mathrm{~mm}$-increment cores taken at breast heigth, and the maximum moisture content method was used for density determinations.

For progenies, 2 samples were cut from each seedling: a slice of $5 \mathrm{~mm}$ in thickness (ref. A) of which the specific gravity was also determined by the maximum moisture content method and a piece of stem of about $35 \mathrm{~mm}$ in length (ref. C) for the specific gravity determination of which the volume was measured with a mercury volumenometer. The densities used for computation were the mean values of the $\mathrm{A}$ and $\mathrm{C}$ densities. Elsewhere another slice of $2 \mathrm{~mm}$ in thickness (ref. B) was X-rayed for fine structure studies of annual rings.

From the mother trees-progenies regression analysis the heritability value was 0.33 , and the correlation coefficient 0.56 .

From the variance analysis within and between progenies, the heritability value was 0.22 , and the $F$ value with family as source of variation was 1.57 significant at a level wery close to $5 \%$.

The relatively low values of heritability were probably due to sampling very small, sizes of the seedlings ( 3 to $9 \mathrm{~mm}$ in diameter) and only one repetition used. 
The figure 1 shows the relationship between the densities of mother-trees and of their progenies.

The figure 2 is an illustration of inheritance: it is a positive print of X-ray photographs 2 times enlarged on which the dense wood is dark. It shows that the denser mother trees $\mathrm{n}^{\circ} 10 \mathrm{~B}$ gives denser seedlings, that the differences arise particularly form springwood and that the densities of the last and that of the last but one rings are closely related in all the seedlings.

The figure 3 deals with the relationship between the density of progenies and the torsion torque measurement made on the mother trees; it shows that the hardness measured in situ during the boring gives a first idea of the density of the seedlings.

Elsewhere in this experience were compared for wood density these progenies (Betouze d'Arques) with the progenies of another stand (Rialsesse) which had itself been obtained from a natural regeneration of the first one. The Betouze d'Arques seedlings had significantly earlier flushing and their wood density were significantly lower.

\section{ZUSAMMENFASSUNG}

\section{DIE HERITABILITAT DER ROHDICHTE DES HOLZES BEI DER WEISSTANNE}

Die Heritabilität der Rohdichte des Holzes wurde an vierjährigen Pflanzen der Weisstanne untersucht, wobei je zehn Nachkommen aus zwanzig freibestäubten Familien verwendet wurden.

Eine Regressionsanalyse zwischen Eltern und Nachkommen ergab einen Heritabilitätswert von 0,33 und einen Korrelationskoeffizienten von 0,$56 ;$ Eine Varianzanalyse zwischen den Familien und innerhalb der Familien ergab einen Heritabilitätswert von 0,22.

Diese relativ niederen Heritabilitätswerte sind voraussichtlich auf die Stichprobennahme zurückzuführen (geringe Abmessungen der Pflanzen - 3 bis $9 \mathrm{~mm}$ Durchmesser - und Probennahme aus einer einzigen Wiederholung).

Es besteht ein signifikanter Zusammenhang zwischen der «Härte» der Mutterbäume und der Rohdichte der Nachkommen im Alter von vier Jahren. Die «Härte» wurde an stehenden Bäumen mit einem Torsionsmesser bestimmt.

Vergleicht man die Rohdichte der Sämlinge von zwei aufeinanderfolgenden Generationen ein und derselben Familie, so kann man eine signifikante Abnahme der Rohdichte bei der zweiten Generation feststellen, welche zweifelsohne mit dem späteren Austreiben der zweiten Generation in Zusammenhang zu bringen ist.

\section{RÉFÉRENCES BIBLIOGRAPHIQUES}

Arbez M., Millier C., 1970. Comparaison de deux générations successives de Sapin (Abies alba Mill.) : structure génétique de population, hypothèses sur l'action de la sélection naturelle. Ann. Sci. forest., 27, (3), 287-301.

Goggans J. F., 1962. The correlation, variation and inheritance of wood properties in Loblolly Pine. School of Forestry, North Carolina State College, Raleigh, Technical report, $n^{\circ} 14$.

Hanson W. D., Robinson H. F., 1963. Statistical genetics and plant breeding. Publ. 982, Nat. Acad. Sci., National Research Council, 623 p.

Lacaze J.F., Polge H., 1970. Relations phénotypiques au stade juvénile entre la densité du bois et diverses caractéristiques phénologiques et de vigueur chez Picea abies Karst. Ann. Sci. forest, 27 (3), 231-242.

Polge H., Illy G., 1968. - Héritabilité de la densité du bois et corrélations avec la croissance étudiées à l'aide de tests non destructifs sur plants de Pins maritimes de quatre ans. Silvae Genetica, 17, Heft 5-6, 173-181, sept.-déc.

Polge H., Keller R., 1970. Première appréciation de la qualité du bois en forêt par utilisation d'un torsiomètre. Ann. Sci. forest., 27 (2), 197-223.

Thiercelin Fr., 1970. Tardiveté du débourrement et densité du bois. Ann. Sci. forest., 27 (3), $243-$ 254.

Squillace A. E., Echolls R. M., Dorman K. W., 1962. Heritability of specific gravity and summerwood percent and relation to other factors in slash pine. Tappi, 45, 599-601.

Stonecypher R., Cech F. C., Zobel B. J., 1964. Inheritance of specific gravity in two- and threeyear-old seedlings of Loblolly pine. Tappi, 47, $405-7$.

Van BuiJtenen J. P., 1962. Heritability estimates of wood density in Loblolly pine. Tappi, 45, 602-5. 